\title{
Article \\ Changes in Triterpenes in Alismatis rhizoma after Processing Based on Targeted Metabolomics Using UHPLC-QTOF-MS/MS
}

\author{
Mengxiang Dai ${ }^{1,+} \oplus$, Sen $\mathrm{Li}^{2,+}{ }^{\dagger}$, Qingxin Shi ${ }^{1}{ }^{\oplus}$, Xingliang Xiang ${ }^{1}$, Yuehui Jin ${ }^{1}$, Sha Wei ${ }^{3}$, Lijun Zhang ${ }^{3}$, \\ Min Yang ${ }^{3}$, Chengwu Song ${ }^{1}$, Rongzeng Huang ${ }^{1, *}$ and Shuna Jin ${ }^{3, *}$ (i)
}

1 College of Pharmacy, Hubei University of Chinese Medicine, 16 Huangjiahu West Road, Wuhan 430065, China; dmx199704@163.com (M.D.); qingxinshi123@hotmail.com (Q.S.); xx1741805629@163.com (X.X.); jinyuehui21@outlook.com (Y.J.); songchengwu2000@163.com (C.S.)

2 Department of Pharmacy, Union Hospital, Tongji Medical College, Huazhong University of Science and Technology, Wuhan 430030, China; lisentome728@hotmail.com

3 College of Basic Medicine, Hubei University of Chinese Medicine, 16 Huangjiahu West Road, Wuhan 430065, China; weisha2021@hbtcm.edu.cn (S.W.); zhanglijun2021@hbtcm.edu.cn (L.Z.); yangmin2021@hbtcm.edu.cn (M.Y.)

* Correspondence: rongzenghuang_2018@hbtcm.edu.cn (R.H.); jinshuna2021@hbtcm.edu.cn (S.J.)

+ These authors contributed equally to this article.

check for updates

Citation: Dai, M.; Li, S.; Shi, Q.; Xiang, X.; Jin, Y.; Wei, S.; Zhang, L.; Yang, M.; Song, C.; Huang, R.; et al. Changes in Triterpenes in Alismatis rhizoma after Processing Based on Targeted Metabolomics Using UHPLC-QTOF-MS/MS. Molecules 2022, 27, 0. https://doi.org/

Academic Editors: René Csuk and Bogusław Buszewski

Received: 22 November 2021 Accepted: 17 December 2021 Published: 23 December 2021

Publisher's Note: MDPI stays neutral with regard to jurisdictional claims in published maps and institutional affiliations.

Copyright: (c) 2021 by the authors. Licensee MDPI, Basel, Switzerland. This article is an open access article distributed under the terms and conditions of the Creative Commons Attribution (CC BY) license (https:// creativecommons.org/licenses/by/ $4.0 /)$.

\begin{abstract}
Alismatis rhizoma (AR) has been used as an herbal medicine in China for over a thousand years. Crude AR, salt-processed AR (SAR), and bran-processed AR (BAR) are recorded in the Pharmacopoeia of the People's Republic of China. However, the differences of chemical composition between crude $\mathrm{AR}$ and its processing products remain limited. In this study, triterpenes were identified from crude AR, SAR, and BAR by ultra-high performance liquid chromatography coupled with quadrupole time-of-flight-mass spectrometer (UHPLC-QTOF-MS/MS). Subsequently, the differences of triterpenes between the crude AR and processed ARs were compared via a targeted metabolomics approach. Finally, a total of 114 triterpenes were identified, of which 83, 100, and 103 triterpenes were found in crude AR, SAR, and BAR, respectively. After salt-processing, there were 17 triterpenes newly generated, 7 triterpenes with trends of increasing, and 37 triterpenes decreased. Meanwhile, 56 triterpenes including 21 newly generated and 35 with significant increases were observed in BAR. This study could be benefit to investigate the processing mechanism of AR, as well as support their clinical applications.
\end{abstract}

Keywords: Alismatis rhizoma; triterpenes; processing; multivariate statistical analysis; metabolomics

\section{Introduction}

In Traditional Chinese Medicine (TCM), processing ("Paozhi" in Chinese) refers to certain types of pharmaceutical technology that promote the use of Chinese herbal medicines to fulfil specific requirements of therapy [1]. Under the guidance of the TCM theory, crude herbs are processed to improve the herb's efficacy and reduce the herb's toxicity and side effects using salt, rice wine, or bran, etc. [2]. Accumulating evidence has shown that along with the contents and structures of constituent's changes, the pharmacological activity of a certain herb usually changes accordingly [2,3]. Thus, the investigation of the changes in the herb's chemical composition after processing is vital to understand the pharmacological mechanism of the herb and its clinical significance.

Alismatis rhizoma (AR, "Zexie" in Chinese), recorded in Chinese Pharmacopoeia (2020 edition), is the dried rhizome of Alisma orientale Juzepzuk, belonging to family Alismataceae [4]. It has been used as a TCM for a long time to treat various diseases such as dysuria, edema, nephropathy, hypertension, and hyperlipidemia [5-7]. Two processed products of AR, salt-processed AR (SAR), and bran-processed AR (BAR), are also recorded in the Chinese Pharmacopoeia Commission [8]. They were usually processed with salt or bran and heated with a certain temperature until their surface appear yellow [9]. Processed 
AR with different methods demonstrates different pharmacological effects in the clinic. Previous studies demonstrated that SAR had stronger diuretic activity than AR, while BAR added the function of tonify the spleen [10]. However, the mechanism for the difference in their efficacy is still unclear.

Pharmacological studies have revealed that triterpenes are the main bioactive constituents responsible for the diuretic, anti-hyperlipidemia, antidiabetic, and anti-inflammatory efficacies of AR [11-14]. A previous study reported that the discrimination of eight triterpenes in AR from different regions [15]. Moreover, recent studies revealed the correlation between the contents of six major triterpenes and the diuretic effect of AR and SAR [16]. However, up to now, quite a few studies have systematically elucidated the influence of different processing methods in the triterpenes between AR, SAR, and BAR. Therefore, exploring the difference in triterpenes between crude AR and processed AR is a prerequisite for understanding their pharmacological mechanisms and clinical significance.

In this study, ultra-high performance liquid chromatography coupled with quadrupole time-of-flight-mass spectrometer (UHPLC-QTOF-MS/MS) was performed to analyze the triterpenes of AR, SAR, and BAR. A targeted metabolomics approach was established to evaluate the changes in triterpenes after processing. Further, the processing mechanism of AR were explored.

\section{Results}

\subsection{Qualitative Analysis of Triterpenes in AR and Processed AR}

The classification of triterpenes was performed according to their structural characteristics and former references. As shown in Figure 1, triterpenes mainly differed in their prevalent oxygenation pattern at the position $C_{16}$. Type I and type II triterpenes had open aliphatic chains at $C_{17}$, while the type III triterpene had a six-membered ring system with an oxygen bridge between $C_{16}$ and $C_{23}$. In contrast to type I triterpenes, type II triterpenes possessed keto groups at $\mathrm{C}_{16}$ in their structure. According to the differences of substitute position, these three types of triterpenes were further divided into eight sub-types. For instance, the type I- 1 triterpene had a hydroxyl or acetyl group substitute at $C_{11}$. The type I-2 triterpene was similar to the type I- 1 except for the substitution of $\mathrm{C}_{16}$. Furthermore, type I-3 triterpenes had no substituent at position 11. Similarly, the differences among the other sub-types of triterpenes can be ascribed to the presence or absence of substituent groups at $\mathrm{C}_{11}, \mathrm{C}_{12}$, and $\mathrm{C}_{16}$.

According to the different structural types, the collision-induced dissociation fragmentation patterns for representative triterpenes of each type skeletons were shown in Figures S1-S8. Based on the type of triterpenes and corresponding fragmentation rules, the triterpenes of the same type could be identified. Triterpenes with a diagnostic product ion at $m / z 339.26$ could be identified as type I- 1 triterpenes. Type I-2 and I-3 triterpenes exhibited a typical ion at $m / z 337.25$ and 341.28 , respectively. In addition, product ion at $m / z 353.25,369.24$, and 355.26 were the characteristic ions of type II-1, II-2, and II-3 triterpenes, respectively. In addition, $m / z 339.27$ and 381.28 were used as the diagnostic product ions of type III- 1 triterpenes, whereas type III-2 triterpenes exhibited diagnostic product ions at $m / z 341.28$ and 383.29. For instance, alisol A 24-acetate was a representative compound of type I-1, and its proposed fragmentation pathway was shown in Figure S1. Alisol A 24-acetate had a $\left[\mathrm{M}+\mathrm{H}-\mathrm{H}_{2} \mathrm{O}\right]^{+}$ion at $m / z 515.3700$ in the full mass spectra scans. The product ions at $m / z 497.35, m / z 455.35$, and $m / z 419.33$ were formed by successive loss of $\mathrm{H}_{2} \mathrm{O}$ and $\mathrm{AcOH}$. The $m / z 383.29$ ion was observed in the product ion related to the dissociation of the $\mathrm{C}_{23}-\mathrm{C}_{24}$ bond, and the product ion at $m / z 365.28$ was acquired by a further loss of $\mathrm{H}_{2} \mathrm{O}$ from the ion of $m / z$ 383.29. In addition, the characteristic fragment at $m / z 339.26$ was obtained by losing $\mathrm{C}_{2} \mathrm{H}_{2}$ from $m / z 365.28$. Therefore, the product ion at $m / z 339.26$ can be used as characteristic diagnostic ions for screening the same type of triterpenes. Similarly, the other types of triterpenes could be identified based on the fragmentation rules and corresponding characteristic product ions. 
Type I

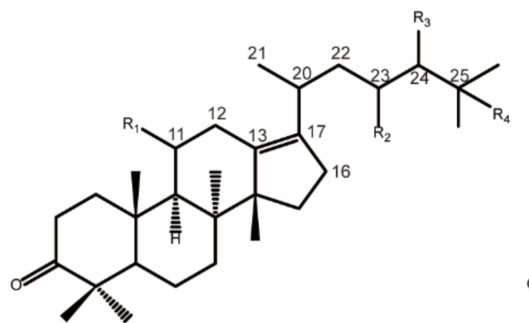

I-1

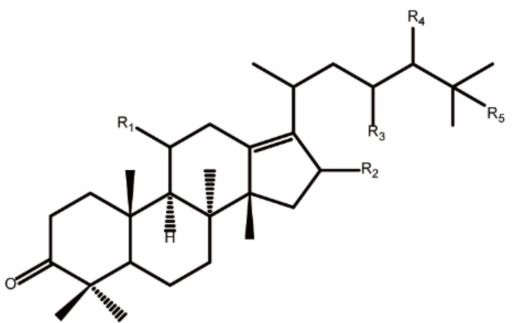

$1-2$<smiles>CC(CC(C)C(N)C(C)(C)C)C1=C2CC[C@]3(C)C(CCC3(C)C)[C@@]2(C)CCC1C(C)(C)C</smiles>

I-3

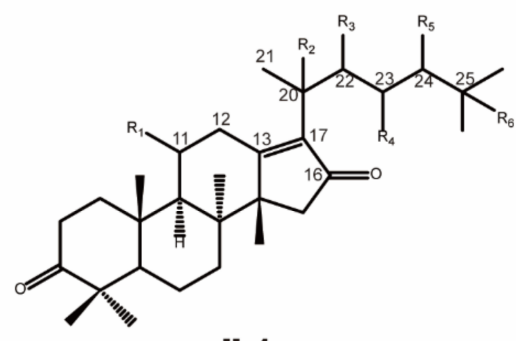

II-1

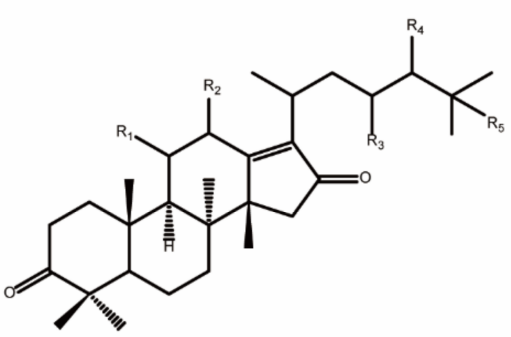

II-2

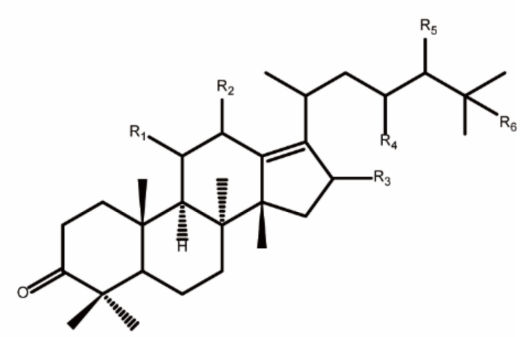

II-3

Typo III

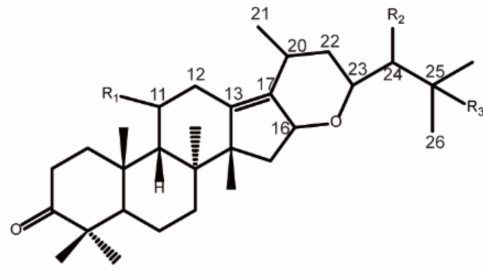

III-1

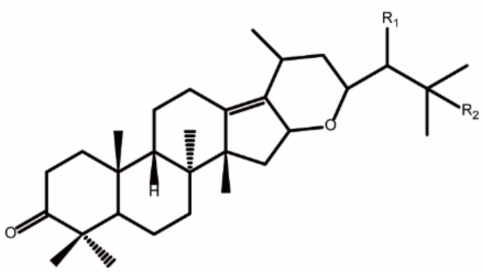

III-2

Figure 1. The major triterpene types in Alismatis rhizoma.

Finally, a total of 114 triterpenes $(83,100$, and 103 compounds in AR, SAR, and BAR, respectively) were identified in AR. Among these triterpenes, 10 were type I- 1 triterpenes, 8 were type I- 2 triterpenes, 6 were type I- 3 triterpenes, 43 were type II- 1 triterpenes, 15 were type II- 2 triterpenes, 6 were type II- 3 triterpenes, 22 were type III- 1 triterpenes, and 4 were type III-2 triterpenes. In addition, the results indicated that 17 new triterpenes were discovered in SAR (Figure 2), including compound 1, 11, 20, 29, 32, 37, 41, 65, 73, 80, 90, 92, 93, 104, 105, 107, and 109. As for BAR, 21 new triterpenes were found in BAR (Figure 2), including compound $3,4,6,8,20,28,29,41,42,45,55,59,67,72,73,90,91,92,98,106$, and 107. Meanwhile, compound 113 which was identified in crude AR disappeared in BAR. The details of the identified triterpenes were summarized in Table S1 and their structures were shown in Table S2.

\subsection{Targeted Metabolomic Analysis of Triterpenes}

A targeted metabolomic analysis was used to compare the triterpenes between AR and processed ARs. In the unsupervised principal component analysis (PCA) model (Figure 3a), the separation was significant $\left(R^{2} X=0.73, Q^{2}=0.666\right)$, which indicated a difference in the levels of triterpenes between crude AR and processed ARs. Thus, the results indicated that different changes in triterpenes had occurred after processing compared with crude AR. The supervised partial least squares-discrimination analyses (PLS-DA) was used to further explore the differences between crude and processed ARs.

As shown in Figure $3 b, c$, the PLS-DA score plots illustrated that the A group was clearly separated from the $\mathrm{S}$ and $\mathrm{B}$ groups. The results of PLS-DA models showed that values of $R^{2} Y$ and $Q^{2}$ were 0.99 and 0.976 for $A$ group and $S$ group, and 0.993 and 0.953 for A group and B group, respectively, which indicates that the models had good abilities for both prediction and reliability. Moreover, the $\mathrm{Q}^{2}$ of the 999 -time permutation tests of the 
aforementioned models were both negative, indicating the two PLS-DA models were not overfitting (Figure S9).
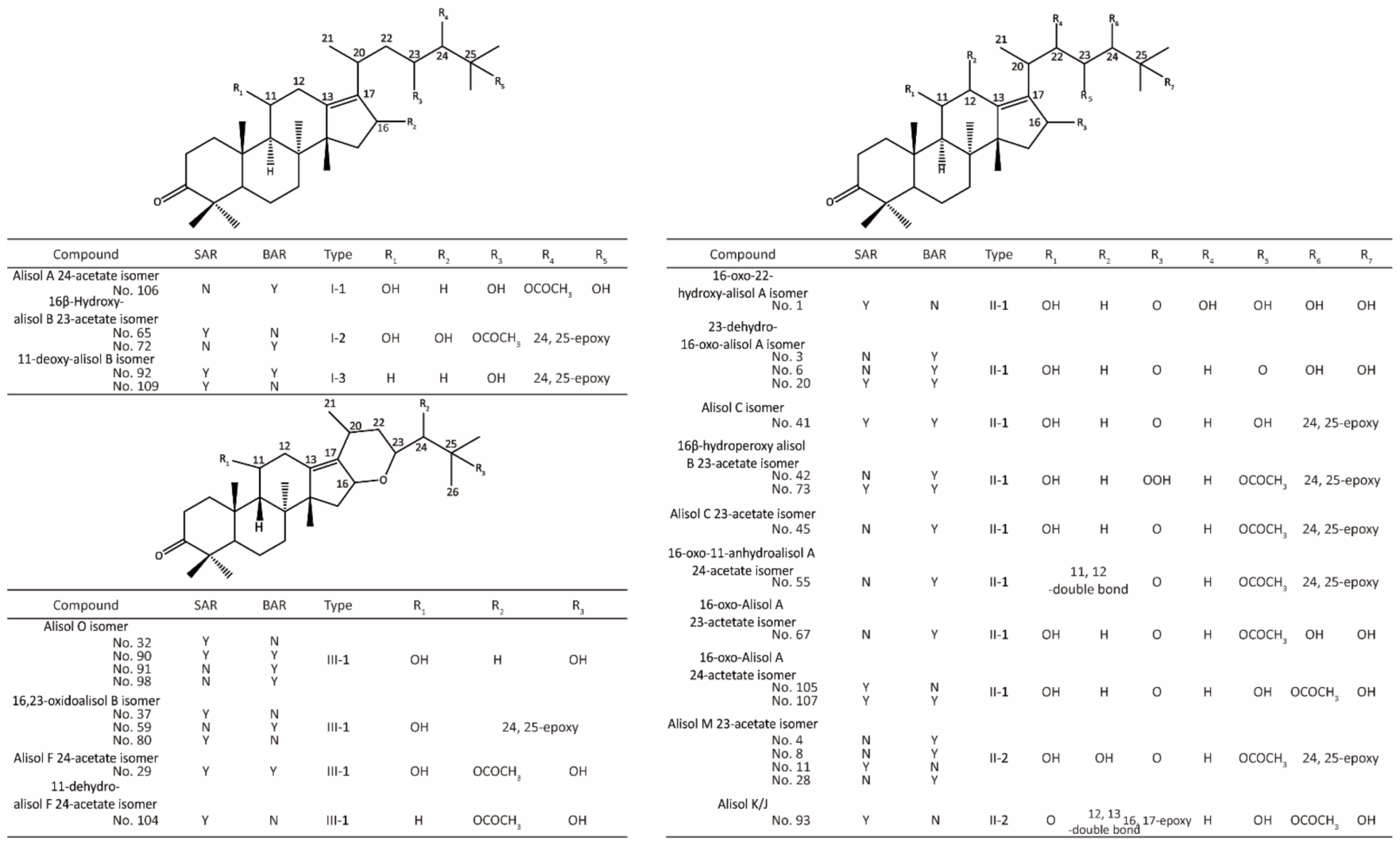

Figure 2. The new generated triterpenes in salt and bran processed AR. N: The compound was not detected in the sample. Y: The compound was detected in the sample.
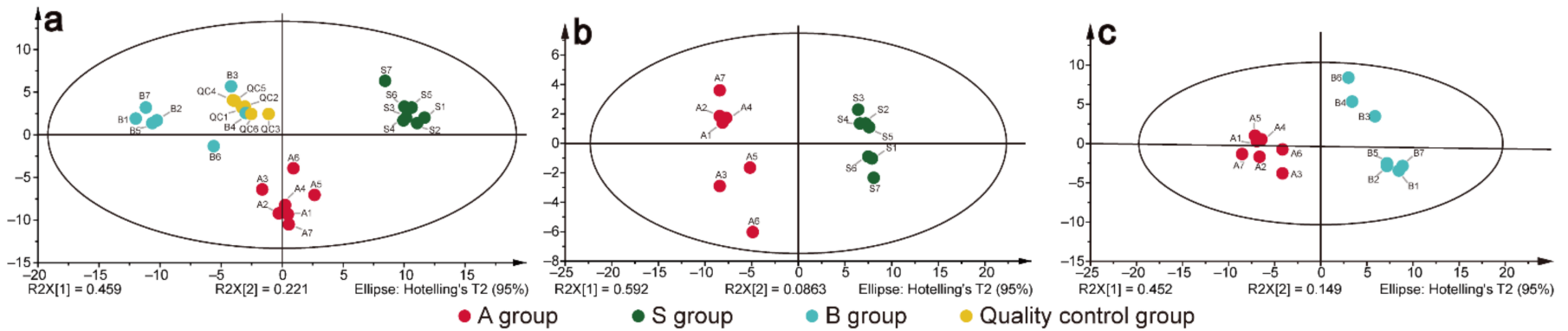

Figure 3. Multivariate statistical analysis of metabolic profiles derived from various AR samples: (a) PCA score scatter plots obtained from the crude samples (group A), salt-processing samples (group S), bran-processing samples (group B) and quality control samples (group QC). (b) PLS-DA score scatter plots obtained from A and S group. (c) PLS-DA score scatter plots obtained from A and B group.

To improve the visualization, these profiles were displayed as heatmap (Figure 4). As depicted in Figure 4, it was observed that most of triterpenes had lower relative contents in the group $S$, while there were no significant differences in the color of the same triterpenes between group A and group B. Some triterpenes were newly generated after processing, for example, compound 1, 3, and 11. The heatmap results provided an overview of the qualitative changes in triterpenes and their variations in relative contents between crude AR and processed ARs. 


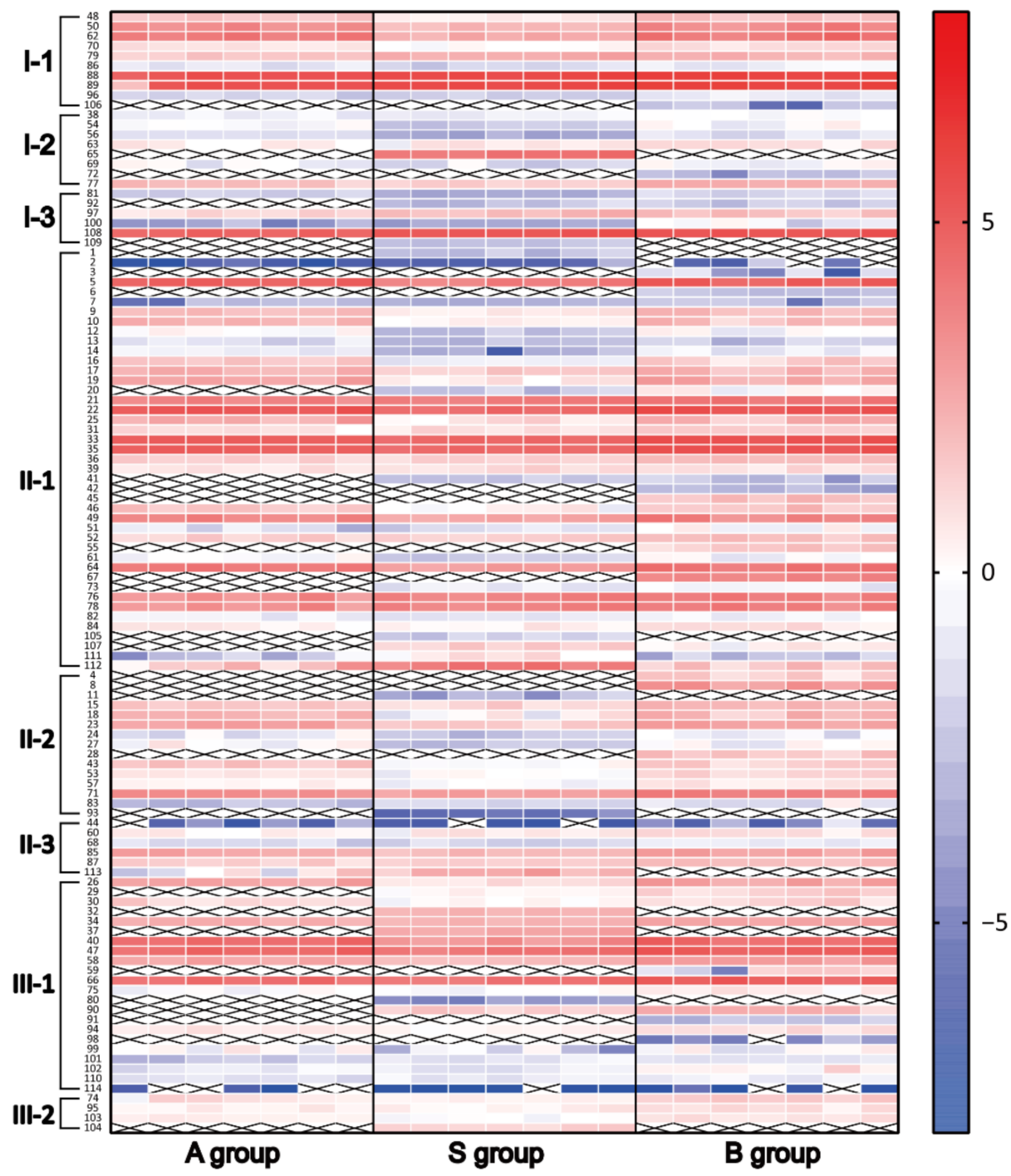

Figure 4. The relative contents of triterpenes detected in the extracts of three samples. A, S, and B are the crude samples, salt-processed samples, and bran-processed samples, respectively. The value of each compound was the $\log 2$ transformation of its relative content. The color intensity from blue to red reflects the relative content of each triterpenes. The triterpenes not detected in the sample were marked as cross in the heatmap.

\subsection{Discovery the Changes in Triterpenes in SAR and BAR}

As depicted in Figure 5, the triterpenes were divided into four groups, including newly generated group, no significance group, decreased group and increased group. According to the PLS-DA analysis (VIP > 1.0) and one-way ANOVA test ( $p$ value $<0.05), 44$ triterpenes occurred significant differences in SAR, including 37 decreased and 7 increased triterpenes. Meanwhile, 35 triterpenes were found to increase significantly in BAR and there were no significant differences in other triterpenes. Compared to crude AR, there were 17 triterpenes newly generated in SAR. The majority of them were type II-1 and type III-1 triterpenes, accounting for 35.29 and $29.41 \%$, respectively. As for BAR, 21 triterpenes newly generated and more than half of them were type II-1 triterpenes (accounting for $61.90 \%$ ). 
a

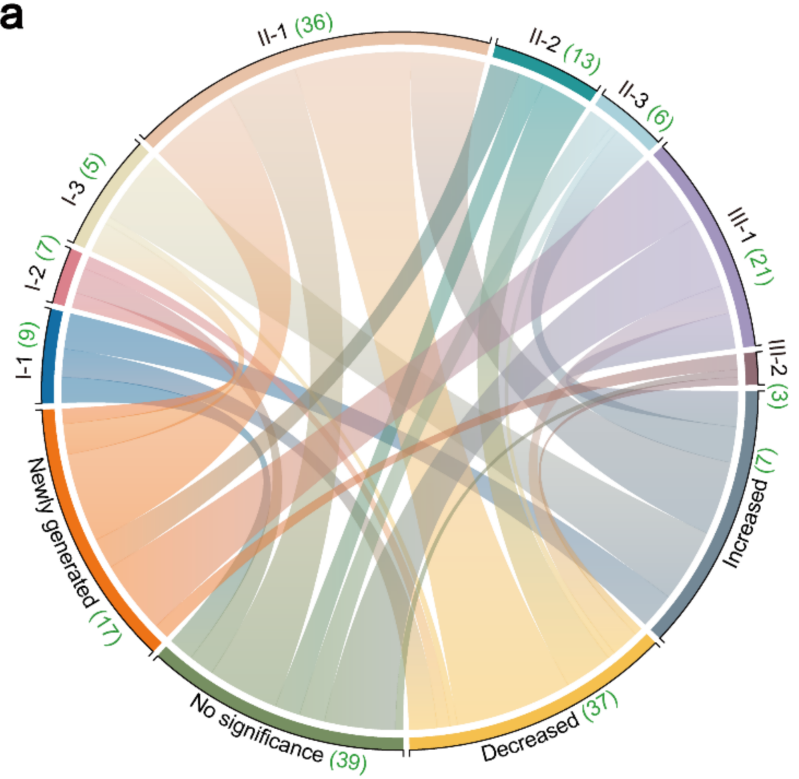

b

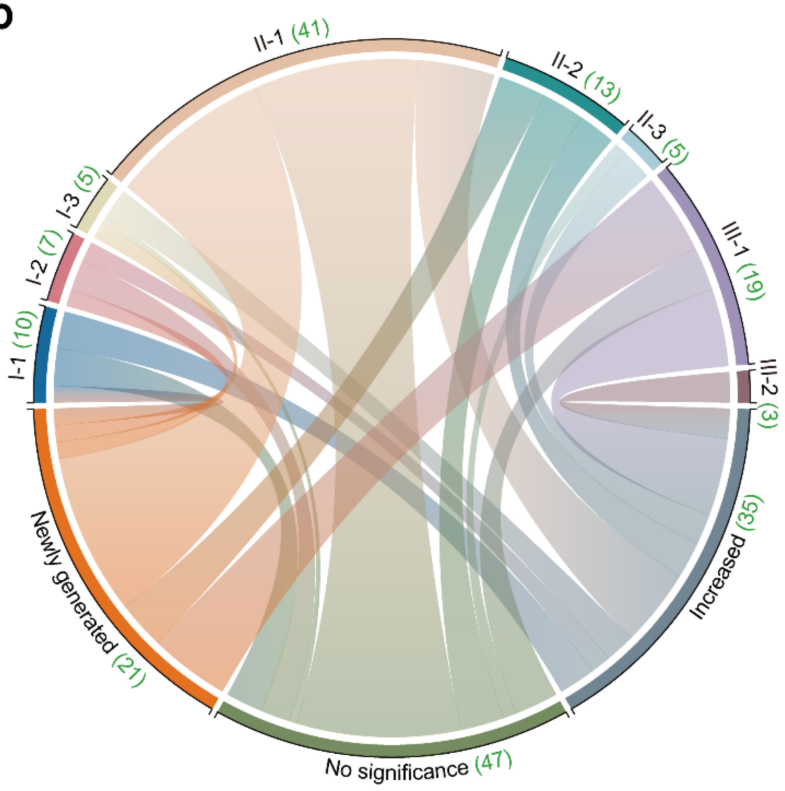

Figure 5. Distribution of triterpenes in the extracts of two processing groups: (a) salt-processing samples. (b) bran-processing samples. The number of different types of triterpenes is indicated by the green number.

Further, the triterpenes with significant differences between AR and its processed products were illustrated as the error line diagram of fold changes based on eight types (see Figure 6). The fold change in a triterpene was the ratio of the mean content of a triterpene in processed AR to that of crude AR. Thus, a fold change value $>1.0$ meant an increased content of the related triterpene after processing, whereas a fold change value $<1.0$ meant a decreased content. It was obvious that all 35 triterpenes with significant differences had an increased in content after bran-processing, while in SAR group, significantly changed triterpenes were mainly decreased in content. 

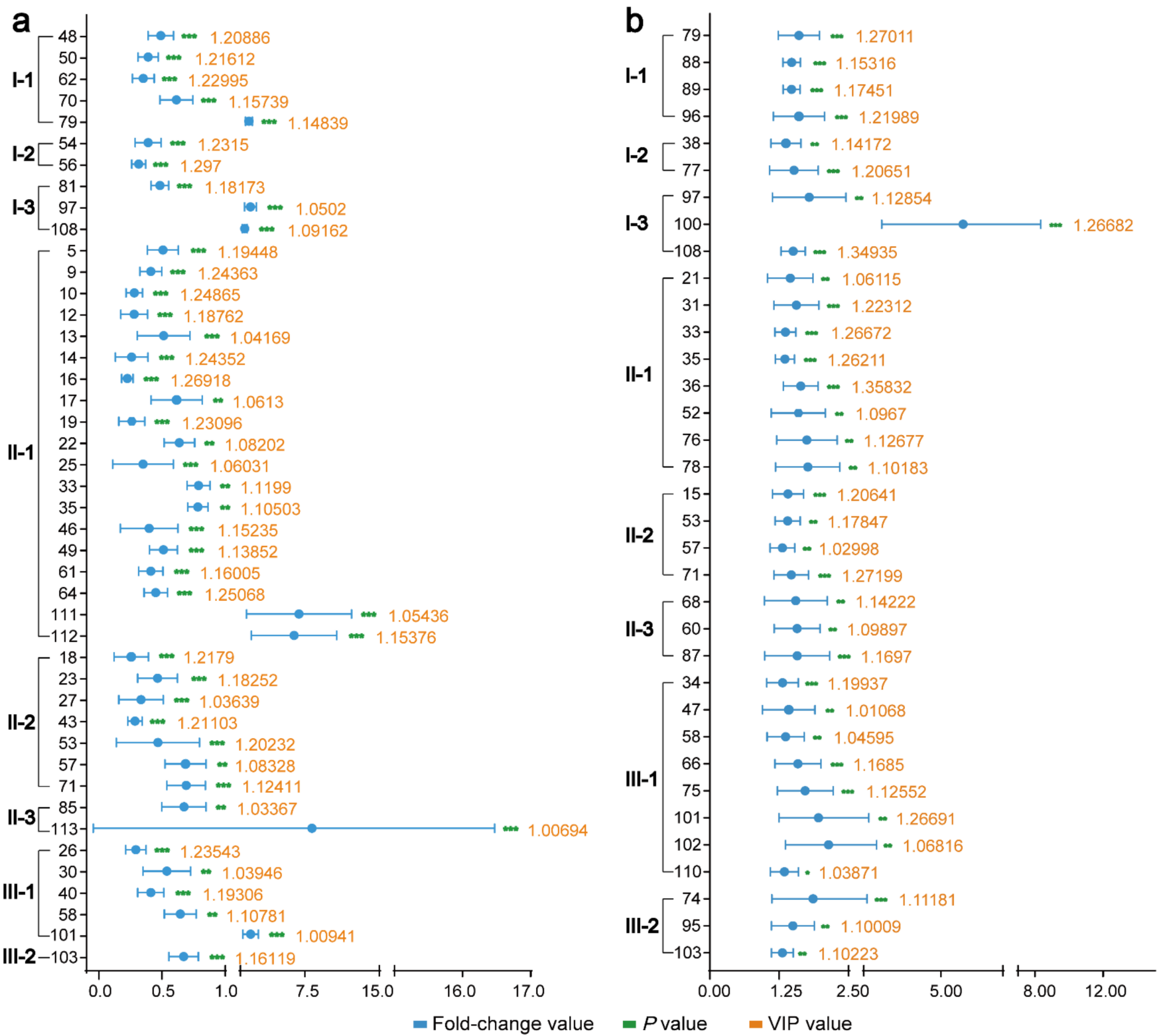

Figure 6. Triterpenes with significant differences between different AR samples: (a) Distribution of triterpenes with significant differences between crude and salt-processed sample based on different structure. (b) Distribution of triterpenes with significant differences between crude and bran-processed sample based on different structure. Values at each point was the ratio of the mean content of a triterpene in processed AR to that of crude AR. Error bars represent the mean \pm S.D. Statistics were performed with one-way ANOVA test, indicated as ${ }^{*} p$ value $<0.05$, ${ }^{* *} p$ value $<0.01$ and ${ }^{* * *} p$ value $<0.001$. The orange number represents the VIP value of the triterpenes.

\section{Discussion}

To explore the differences between crude and processed ARs, the UHPLC-QTOFMS/MS combined with multivariate statistical analysis was developed to identify and distinguish the difference in triterpenes in crude AR and processed ARs. In this study, a total of 83,100 , and 103 triterpenes were identified in crude AR, SAR, and BAR, respectively. There were 17 and 21 newly generated triterpenes were detected in SAR and BAR. Meanwhile, compared with the content of triterpenes in crude AR, 44 triterpenes had significantly changed after salt-processing, including 37 decreased and 7 increased triterpenes. While after bran-processing, 35 triterpenes were significant differences and all of them showed an increase in contents. 
Many methods of processing, such as stir-frying, steaming, and boiling, need to be carried out at the heating conditions, which probably leads to complex chemical changes [17]. In this study, AR was processed with salt water under the temperature range of 80 to $120^{\circ} \mathrm{C}$. Our results showed that in SAR, compounds 14, 17, 18, 19, 22, 23, 25, 27, 33, 35, 43, $46,49,53,57,64,71$, and 75 , which all possessed a $C_{24}, C_{25}$-oxide three-membered ring of the side chain were massively reduced in type II triterpenes compared to type I triterpenes. It was speculated that the type II triterpenes with a $\mathrm{C}_{24}, \mathrm{C}_{25}$-oxide three-membered ring of the side chain were more unstable than others. A previous study indicated that owing to the presence of a carbonyl group at $C_{16}$, cracking and ring opening of triterpenes at position 24 and 25 was more easily than others [18]. In addition, the contents of type I (compound 48, 50, 54, 56, 62, 70, and 81) and type III (compound 26, 30, 40, 58, and 103) triterpenes with hydroxyl or acetyl groups at $C_{23}, C_{24}$, and $C_{25}$ were significantly reduced after salt-processing. It was reported that the compounds with substituting groups, such as hydroxyl and acetyl groups, would lose $\mathrm{H}_{2} \mathrm{O}$ and $\mathrm{AcOH}$ under high temperature [16,19]. Moreover, there was the evidence suggesting that the total contents of triterpenes were decreased with the baking temperature increasing, especially when the temperature was above $80{ }^{\circ} \mathrm{C}[20]$.

However, the reduction in certain triterpenes after heating was not observed in BAR, on the contrary, triterpenes with significant differences showed an increasing trend after bran-processing. Our findings indicated that triterpenes in crude AR had different change trends after two processing of SAR and BAR. In this study, AR was processed at two methods with different excipients (salt and bran), temperature ranges ( 80 to $120{ }^{\circ} \mathrm{C}$ and 150 to $220^{\circ} \mathrm{C}$ ) and heating durations (around 30-40 $\mathrm{min}$ and around $8 \mathrm{~min}$ ); thus, these parameters might be responsible for the different change trends of triterpenes between SAR and BAR. In addition to the heating temperature, heating duration was also an important factor in AR processing. A report about triterpenes suggested that the contents of alisol A 24-acetate and alisol B 23-acetate initially increased as the duration of heating time, but after 40 min of heating, this increasing tendency ceased and the concentrations rapidly decreased [21]. Considering the heating duration of around $8 \mathrm{~min}$ for bran-processing in our study, the increase in triterpene contents in BAR were consistent with the previous report. Meanwhile, emerging evidence has indicated that under heating condition, excipients used in processing could often help active constituents to dissolve more easily from a complex texture [22,23]. The existing of bran made sample heated evenly, and the heat duration of BAR was much shorter than SAR. Taken together, the current study demonstrated that the heating temperature, heating duration, and excipient were the crucial factors in AR processing.

For the effectiveness of TCM, herbal may profit from synergistic functions of multiple chemical ingredients [24,25]. Although most of triterpene's contents were decreased, there were some triterpene contents that increased and newly formed after salt-processing. For example, alisol A (compound 79), 11-deoxy-alisol B (compound 97), 11, 25-anhydro-alisol F (compound 101), and 11-deoxy-alisol B 23 acetate (compound 108) were found with significance increases after processed with salt. In addition, the therapeutic efficacy of SAR might benefit from synergistic actions of multiple ingredients. During the processing, complicated changes in herbal components may occur, and the pharmacological activity of a certain herbal medicine may be changed accordingly [1]. Previous studies demonstrated that SAR showed a better hepatoprotective activity and diuretic effect than crude AR $[16,26]$. This phenomenon also occurs in other salt-processed plant medicine $[27,28]$.

Generally, the main purposes of processing are reducing toxicity and enhancing the effects of crude drugs. Previous studies indicated that the hydroxyl and acetyl groups on the side chain of triterpene were the essential groups to exert lipid-lowering effect [29,30]. For example, alisol A (compound 79) is one of the main active triterpenes in AR and possesses many biological activities including antibacterial, antiviral, lipid regulating, and diuretic effects [16,31-33]. It showed a similarity in the pattern of content changes in SAR and BAR (the fold change value of SAR and BAR was 1.849 and 1.566, respectively). Furthermore, it 
was reported that triterpenes with the carbonylation on the $\mathrm{C}_{16}$ of the parent nucleus and a $\mathrm{C}_{24}, \mathrm{C}_{25}$-oxide three-membered ring of the side chain had relation with the lipid-lowering activity [9]. In this study, among the newly generated and increased triterpenes, most of them showed similar characteristic features, which were a $\mathrm{C}_{24}, \mathrm{C}_{25}$-oxide three-membered ring of the side chain no matter in SAR or BAR. Moreover, processing Chinese medicine can enhance the efficacy and change the trend of action [1,34]. The difference in changing trends of triterpene's contents between SAR and BAR might bring out potential changes in pharmacological activity. Since salt-processing could enhance the effect of AR in diuretic activity [16], the contents increased in BAR might reinforce the activity of AR. Results of a previous study indicated that the diuretic effect of BAR was better than SAR [18]. In addition, there was the evidence showed that SAR exhibited better hepatoprotective effects than crude and bran-processed AR by lowering levels of serum AST and ALT [35]. In conclusion, the effects of heating, heating durations, and excipients in the processing procedure might be the main causes of the changes in chemical composition, as well as the subsequent impacts on the efficacy of crude AR.

\section{Materials and Methods}

\subsection{Chemicals and Reagents}

Acetonitrile (ACN, LC-MS grade) and formic acid ( $\geq 98 \%$, analytical grade) were purchased from Fisher Scientific (Fair Lawn, NJ, USA) and Sinopharm Chemical Reagent Co., Ltd. (Shanghai, China), respectively. Purified water was obtained from Millipore using a Milli-Q system (Bedford, MA, USA). The dried rhizomes of cultivated AR were collected from Sichuan province of China in April 2019 and identified by Prof. Keli Chen of Hubei University of Chinese Medicine. The dried rhizomes of AR were cut into 2-4 mm slices before used.

The reference standards (alisol F, alisol F-24-acetate, 24-deacetyl alisol O, alisol A 24-acetate, alisol B 23-acetate and alisol C 23-acetate) were previously isolated and purified from the dried rhizome of AR. The purities of these standards were tested to be above $98 \%$ using HPLC coupled with UV detector.

\subsection{Preparation of $S A R$ and $B A R$}

SAR and BAR were prepared according to the procedure recorded in the general principles of Chinese pharmacopoeia (2020 edition) [36]. Briefly, the crude AR slices (1 kg) was mixed and soaked with $2 \%$ salt solution $(2: 100$, salt-water, $w / v)$ until fully absorbed in a sealed container. Then the moistened AR were stir-fried in a metallic pan over a low flame $\left(80-120^{\circ} \mathrm{C}\right)$ around $30-40 \mathrm{~min}$ until the water evaporated. Similarly, the bran (100 g) was placed in a preheated stir-frying pan. When the bran was fuming, added $1 \mathrm{~kg}$ of AR and stirred rapidly around 8 min until the color of AR turned yellow, and removed the bran. After cooling to room temperature, SAR and BAR were obtained and used for further analysis.

\subsection{Preparation of Sample Solutions}

Seven batches of AR were smashed and sieved (24 mesh) to obtain homogeneous powders separately. Sample powder $(0.5 \mathrm{~g})$ was ultrasonically extracted with $5 \mathrm{~mL}$ acetonitrile at room temperature for $30 \mathrm{~min}$. Then, the supernatant of these samples was centrifuged at $14,000 \mathrm{rpm}$ for $10 \mathrm{~min}$ followed by filtration, after which $5 \mu \mathrm{L}$ of supernatant of each sample with fexofenadine $\left(100 \mathrm{ng} \cdot \mathrm{mL}^{-1}\right)$ as internal standard was injected and analyzed by the UHPLC-QTOF-MS/MS. SAR and BAR were extracted with the same procedure as above. Thus, the samples were separated into 3 groups: raw AR group (A group), salt-processed AR group (S group), and bran-processed AR group (B group). Meanwhile, the quality control samples (QC) were prepared by pooling each solution from all samples. To assess of the stability of analytical systems, the QC samples were performed at the beginning, the end, and every 5 injections throughout the whole UHPLC-MS/MS analysis. 


\subsection{Chromatography and Mass Spectrometric Analysis}

UHPLC-MS/MS analysis was performed on a Waters ACQUITY Ultra Performance LC system. A C18 column (100 mm × $2.1 \mathrm{~mm}$ i.d., $1.7 \mu \mathrm{m}$, Waters, Milford, MA, USA) was used for separation. The flow rate was $0.3 \mathrm{~mL} \cdot \mathrm{min}^{-1}$ and the injection volume was $5 \mu \mathrm{L}$ with a column temperature at $40{ }^{\circ} \mathrm{C}$. The mobile phase of the eluent was $0.1 \%$ formic acid in water (A) and acetonitrile (B), and the gradient conditions were as follows: $0-18 \mathrm{~min}$, 20-77\% B; 18-25 min, 77-95\% B; 25-27 min, 95\% B; 27-28 min, 95-20\% B; 28-30 min, 20\% B.

Mass spectrometric analyses were performed on a Waters Xevo G2-XS QTof system with an electrospray ionization source and the positive ion mode was used for data acquisition. The following MS conditions were set: cone gas flow, $50 \mathrm{~L} \cdot \mathrm{h}^{-1}$; desolvation gas flow, $600 \mathrm{~L} \cdot \mathrm{h}^{-1}$; desolvation temperature, $500{ }^{\circ} \mathrm{C}$; source temperature, $100{ }^{\circ} \mathrm{C}$; cone voltage, $60 \mathrm{~V}$; capillary voltage, $3 \mathrm{kV}$. The collision energy was set at $30 \mathrm{eV}$ and the collision energy spread was $40 \mathrm{eV}$ for MS/MS experiments. The mass ranges were set at $m / z 100-1200$ for full scan with a scan duration of $1 \mathrm{sec}$.

\subsection{Statistics Analysis}

The raw spectra data were acquired and managed using MassLynx software (Waters Corporation, Milford, MA, USA). Quantitative results were obtained by dividing the peak area of each triterpene by the peak area of the internal standard fexofenadine. After this procedure, the resulting dataset was on multivariate statistical analysis and one-way ANOVA using the SIMCA-P software (Umetrics AB, Umea, Sweden, version 14.1) and GraphPad Prism (GraphPad Software, La Jolla, CA, USA).

To evaluate the differentiation of chemical compounds caused by different processing methods, crude AR, SAR, and BAR were set as groups A, S, and B, respectively. QC samples were set as group QC and used to evaluate the repeatability and stability of the whole sample set [37]. To determine whether the triterpenes in extract of processed AR differed from those of the crude AR, PCA was used for multivariate exploration of clusters and trends among the four groups. Subsequently, using PLS-DA, an examination for the differences of triterpenes between AR samples was achieved. Moreover, permutation tests (999 cycles) were conducted to assess model overfitting. The cross-validation parameters $R^{2} Y$ and $Q^{2}$ were calculated to evaluate the goodness-of-fit and predictive ability of PLS-DA model. Variable importance in the projection (VIP) was a parameter that showed the importance of a variable in a model. Usually, a compound with VIP $>1.0$ was considered to play an important role in distinguishing groups $[37,38]$. Triterpenes that met the following two criteria were regarded as the differential metabolites between crude AR and process ARs. First, VIP values in PLS-DA model were greater than 1.0. The other was that the contents of triterpenes had significant differences $(p$ value $<0.05)$ calculated by one-way ANOVA.

\section{Conclusions}

In this study, UHPLC-QTOF-MS/MS combined with a targeted metabolomics analysis was applied to investigate the changes in triterpenes between crude AR and processed ARs. As a result, a total of 83, 100, and 103 triterpenes were identified in crude AR, SAR, and BAR, respectively. Except for the newly generated triterpenes, there were 44 and 35 triterpenes were changed significantly after salt and bran processing. Our results suggested that triterpenes in crude AR had different change trends after two processing of SAR and BAR. In SAR group, significantly changed triterpenes were mainly decreased in content, while all 35 triterpenes with significant differences had an increased in content after bran-processing. The different change trends between SAR and BAR could be attributed to different heating durations, temperatures, and excipients. The results of this study could provide scientific evidences for the variations of triterpenes in AR under different processing methods, and be valuable to elucidate the processing mechanisms of AR. Further studies will focus on the relationship between the differences of triterpene and pharmacological activity after AR processing. 
Supplementary Materials: The following supporting information can be downloaded online. Figure S1: The proposed fragmentation pathway of alisol A 24-acetate, Figure S2: The proposed fragmentation pathway of 16 $\beta$-Hydroxy-alisol B 23-acetate, Figure S3: The proposed fragmentation pathway of 11-deoxy-alisol B, Figure S4: The proposed fragmentation pathway of alisol C 23-acetate, Figure S5: The proposed fragmentation pathway of alisol M 23-acetate, Figure S6: The proposed fragmentation pathway of 11-Deoxy-alisol C 23-acetate, Figure S7: The proposed fragmentation pathway of alisol O, Figure S8: The proposed fragmentation pathway of alisol I, Figure S9: Goodness of fit and validations (permutation tests) of the PLS-DA models. (a) scoring plots for 999-time permutation validation test for PLS-DA models that corresponded to the raw sample and salt-processed sample. (b) scoring plots for 999-time permutation validation test for PLS-DA models that corresponded to the raw sample and bran-processed sample, Table S1: Identification of triterpenes obtained from the extract of Alismatis rhizoma using UHPLC-QTOF-MS/MS, Table S2: Chemical structures of identified triterpenes from Alismatis rhizoma.

Author Contributions: M.D. performed the data analyses and wrote the original draft. S.L. interpreting the results and revised the original draft. Q.S., X.X., Y.J., S.W., L.Z., M.Y. and C.S. conducted the experiments and interpreted the results. R.H. and S.J. developed the concept, designed the study, and revised the manuscript. All authors have read and agreed to the published version of the manuscript.

Funding: This research was funded by National Natural Science Foundation of China, grant number 81803717.

Institutional Review Board Statement: Not applicable.

Informed Consent Statement: Not applicable.

Data Availability Statement: The datasets generated and/or analyzed during the current study are available from the corresponding author on reasonable request.

Conflicts of Interest: The authors declare no conflict of interest.

Sample Availability: Samples of the crude Alismatis rhizoma, salt-processed Alismatis rhizoma and bran-processed Alismatis rhizoma are available from the authors.

$\begin{array}{ll}\text { Abbreviations } & \\ \text { TCM } & \text { traditional Chinese Medicine; } \\ \text { AR } & \begin{array}{l}\text { Alismatis rhizoma; } \\ \text { salt-processed AR; } \\ \text { SAR }\end{array} \\ \text { bAran-processed AR; } \\ \text { UHPLC-QTOF-MS/MS } & \text { ultra-high performance liquid chromatography coupled with } \\ & \text { quadrupole time-of-flight-mass spectrometer; } \\ \text { PCA } & \text { principal component analysis; } \\ \text { PLS-DA } & \text { partial least squares-discrimination analyses; } \\ \text { VIP } & \text { variable importance in the projection; } \\ \text { One-way ANOVA } & \text { one-way analysis of variance; } \\ \text { A group } & \text { raw AR group; } \\ \text { S group } & \text { salt-processed AR group; } \\ \text { B group } & \text { bran-processed AR group; } \\ \text { QC } & \text { quality control samples. }\end{array}$

\section{References}

1. Wu, X.; Wang, S.; Lu, J.; Jing, Y.; Li, M.; Cao, J.; Bian, B.; Hu, C. Seeing the unseen of Chinese herbal medicine processing (Paozhi): Advances in new perspectives. Chin. Med. 2018, 13, 4. [CrossRef]

2. Zhao, Z.; Liang, Z.; Chan, K.; Lu, G.; Lee, E.; Chen, H.; Li, L. A unique issue in the standardization of Chinese materia medica: Processing. Planta Med. 2010, 76, 1975-1986. [CrossRef]

3. Jiang, H.; Yang, L.; Hou, A.; Zhang, J.; Wang, S.; Man, W.; Zheng, S.; Yu, H.; Wang, X.; Yang, B.; et al. Botany, traditional uses, phytochemistry, analytical methods, processing, pharmacology and pharmacokinetics of Bupleuri Radix: A systematic review. Biomed. Pharmacother. 2020, 131, 110679. [CrossRef]

4. Shu, Z.; Pu, J.; Chen, L.; Zhang, Y.; Rahman, K.; Qin, L.; Zheng, C. Alisma orientale: Ethnopharmacology, Phytochemistry and Pharmacology of an Important Traditional Chinese Medicine. Am. J. Chin. Med. 2016, 44, 227-251. [CrossRef] 
5. Zhang, X.; Li, X.; Lin, N.; Zhao, W.; Huang, X.; Chen, Y.; Huang, M.; Xu, W.; Wu, S. Diuretic Activity of Compatible Triterpene Components of Alismatis rhizoma. Molecules 2017, 22, 1459. [CrossRef] [PubMed]

6. Chu, Y.; Jiang, H.; Ju, J.; Li, Y.; Gong, L.; Wang, X.; Yang, W.; Deng, Y. A metabolomic study using HPLC-TOF/MS coupled with ingenuity pathway analysis: Intervention effects of Rhizoma Alismatis on spontaneous hypertensive rats. J. Pharm. Biomed. Anal. 2016, 117, 446-452. [CrossRef]

7. Miao, H.; Zhang, L.; Chen, D.; Chen, H.; Zhao, Y.; Ma, S. Urinary biomarker and treatment mechanism of Rhizoma Alismatis on hyperlipidemia. Biomed. Chromatogr. 2017, 31, e3829. [CrossRef] [PubMed]

8. The State Pharmacopoeia Commission of PRC. Pharmacopoeia of the People's Republic of China; Chinese Medical Science and Technology Press: Beijing, China, 2020; Volume 1, p. 239.

9. Li, S.; Wang, L.; Du, Z.; Jin, S.; Song, C.; Jia, S.; Zhang, Y.; Jiang, H. Identification of the lipid-lowering component of triterpenes from Alismatis rhizoma based on the MRM-based characteristic chemical profiles and support vector machine model. Anal. Bioanal. Chem. 2019, 411, 3257-3268. [CrossRef]

10. Liu, D.; Gong, Q.; Liu, Q.; Su, P.; Wang, J.; Zhong, L.; Zhang, J.; Hao, G.; Yu, H.; Wang, Y. Overview of Research on Harvest, Processing in Production Place, Processing and Quality Evaluation of Alismatis Rhizoma. Chin. J. Exp. Tradit. Med. Formulae 2018, 24, 203-211. [CrossRef]

11. Liu, S.; Sheng, W.; Li, Y.; Zhang, S.; Zhu, J.; Gao, H.; Yan, L.; Wang, Z.; Gao, L.; Zhang, M. Chemical constituents from Alismatis Rhizoma and their anti-inflammatory activities in vitro and in vivo. Bioorg. Chem. 2019, 92, 103226. [CrossRef] [PubMed]

12. Feng, Y.; Chen, H.; Tian, T.; Chen, D.; Zhao, Y.; Lin, R. Diuretic and anti-diuretic activities of the ethanol and aqueous extracts of Alismatis rhizoma. J. Ethnopharmacol. 2014, 154, 386-390. [CrossRef] [PubMed]

13. Jang, M.; Han, Y.; Nam, J.; Han, C.; Kim, B.; Jeong, H.; Ha, K.; Jung, M. Protective Effects of Alisma orientale Extract against Hepatic Steatosis via Inhibition of Endoplasmic Reticulum Stress. Int. J. Mol. Sci. 2015, 16, 26151-26165. [CrossRef]

14. Lau, C.; Chan, C.; Chan, Y.; Lau, K.; Lau, T.; Lam, F.; Che, C.; Leung, P.; Fung, K.; Ho, Y.; et al. In vitro antidiabetic activities of five medicinal herbs used in Chinese medicinal formulae. Phytother. Res. 2008, 22, 1384-1388. [CrossRef]

15. Tian, S.; Zhao, X.; Liu, S.; Feng, W.; Chen, L.; Yan, L.; Wang, Z. Regional differences analysis of Alismatis Rhizoma based on UPLC characteristic chromatogram and determination of eight terpenoids. Zhongguo Zhong Yao Za Zhi 2020, 45, 1545-1557. [CrossRef]

16. Tao, Y.; Jiang, E.; Yan, J.; Cai, B. A biochemometrics strategy for tracing diuretic components of crude and processed Alisma orientale based on quantitative determination and pharmacological evaluation. Biomed. Chromatogr. 2020, 34, e4744. [CrossRef]

17. Chen, Z.; Ye, S.; Zhu, R. The extraordinary transformation of traditional Chinese medicine: Processing with liquid excipients Pharm. Biol. 2020, 58, 561-573. [CrossRef]

18. Yan, G.; Lan, M.; Qiu, J.; Li, X.; Xu, W.; Wu, S. Study on Changes of Chemical Composition and Diuretic Effect in Alismatis Rhizoma after Processing. Chin. J. Inf. TCM 2020, 27, 59-65. [CrossRef]

19. Li, S.; Jin, S.; Song, C.; Jia, S.; Zhang, Y.; Feng, Y.; Du, Z.; Jiang, H. The strategy for establishment of the multiple reaction monitoring based characteristic chemical profile of triterpenes in Alismatis rhizoma using two combined tandem mass spectrometers J. Chromatogr. A 2017, 1524, 121-134. [CrossRef]

20. Tai, Y.; Zou, F.; Zhang, Q.; Wang, J.; Rao, R.; Xie, R.; Wu, S.; Chu, K.; Xu, W.; Li, X.; et al. Quantitative Analysis of Eight Triterpenoids and Two Sesquiterpenoids in Rhizoma Alismatis by Using UPLC-ESI/APCI-MS/MS and Its Application to Optimisation of Best Harvest Time and Crude Processing Temperature. J. Anal. Methods Chem. 2019, 2019, 8320171. [CrossRef] [PubMed]

21. Dai, X. Research on the Processing Technology and Function of Alisma Orientale. Master's Thesis, Liaoning University of Chinese Medicine, Shenyang, China, 2009.

22. Liu, F.; Zhang, Z.; Lai, J.; Hu, B. Determination of four kinds of alkaloids from Rhizoma Coptis and processed Rhizoma Coptis by HPLC. Chin. Tradit. Patent Med. 2010, 32, 1925-1928.

23. Zhan, J.; Zheng, K.; Zhu, K.; Bi, C.; Zhang, W.; Du, C.; Fu, Q.; Dong, T.; Choi, R.; Tsim, K.; et al. Chemical and biological assessment of Angelicae Sinensis Radix after processing with wine: An orthogonal array design to reveal the optimized conditions. J. Agric. Food Chem. 2011, 59, 6091-6098. [CrossRef] [PubMed]

24. Yang, Y.; Zhang, Z.; Li, S.; Ye, X.; Li, X.; He, K. Synergy effects of herb extracts: Pharmacokinetics and pharmacodynamic basis. Fitoterapia 2014, 92, 133-147. [CrossRef] [PubMed]

25. Caesar, L.; Cech, N. Synergy and antagonism in natural product extracts: When $1+1$ does not equal 2. Nat. Prod. Rep. 2019, 36, 869-888. [CrossRef]

26. Song, L.; Pan, R.; Tian, X.; He, M. Effect of salt-processing on anti fatty liver effect of Alismatis rhizoma. Lishizhen Med. Mater. Med. Res. 2018, 29, 92-94. [CrossRef]

27. Lu, J.; Liu, L.; Zhu, X.; Wu, L.; Chen, Z.; Xu, Z.; Li, W. Evaluation of the Absorption Behavior of Main Component Compounds of Salt-Fried Herb Ingredients in Qing'e Pills by Using Caco-2 Cell Model. Molecules 2018, 23, 3321. [CrossRef]

28. Wang, H.; Li, W.; Gao, Q.; Weng, Z.; Zhao, G.; Chen, Z.; Cai, B. Study on Contents of Twelve Indicative Components in Qing'e Pills Composed of Herbs Processed by Different Methods. Tradit. Chin. Drug Res. Clin. Pharmacol. 2016, 27, 684-688. [CrossRef]

29. Imai, Y.; Matsumura, H.; Aramaki, Y. Hypocholesterolemic effect of alisol A-24-monoacetate and its related compounds in rats Jpn. J. Pharmacol. 1970, 20, 222-228. [CrossRef] [PubMed]

30. Xu, F.; Yu, H.; Lu, C.; Chen, J.; Gu, W. The Cholesterol-Lowering Effect of Alisol Acetates Based on HMG-CoA Reductase and Its Molecular Mechanism. Evid. Based Complement. Alternat. Med. 2016, 2016, 4753852. [CrossRef] 
31. Ma, Q.; Han, L.; Bi, X.; Wang, X.; Mu, Y.; Guan, P.; Li, L.; Huang, X. Structures and biological activities of the triterpenoids and sesquiterpenoids from Alisma orientale. Phytochemistry 2016, 131, 150-157. [CrossRef]

32. Zhang, Q.; Jiang, Z.; Luo, J.; Ma, Y.; Liu, J.; Guo, R.; Zhang, X.; Zhou, J.; Niu, W.; Du, F.; et al. Anti-HBV agents. Part 3: Preliminary structure-activity relationships of tetra-acylalisol A derivatives as potent hepatitis B virus inhibitors. Bioorg. Med. Chem. Lett. 2009, 19, 6659-6665. [CrossRef]

33. Xu, F.; Chen, J.; Zhang, Y.; Wu, Q.; Shen, Y.; Gu, W.; Liu, S.; Lu, C.; Liao, H.; Bao, K. Molecular insight into the mechanism of lipid regulating effect of Alisma orientalis based on ACAT. Int. J. Biol. Macromol. 2020, 158, 1141-1162. [CrossRef]

34. Xia, F.; Liu, C.; Wan, J. Characterization of the cold and hot natures of raw and processed Rehmanniae Radix by integrated metabolomics and network pharmacology. Phytomedicine 2020, 74, 153071. [CrossRef] [PubMed]

35. Chen, X.; Li, H. Hepatoprotective effect of Alismatis rhizoma and different processed products on acute liver injury in mice. J. Chin. Med. Mater. 2006, 29, 592-593. [CrossRef]

36. The State Pharmacopoeia Commission of PRC. Pharmacopoeia of the People's Republic of China; Chinese Medical Science and Technology Press: Beijing, China, 2020; Volume 4, pp. 31-32.

37. Li, Y.; Jin, Y.; Yang, S.; Zhang, W.; Zhang, J.; Zhao, W.; Chen, L.; Wen, Y.; Zhang, Y.; Lu, K.; et al. Strategy for comparative untargeted metabolomics reveals honey markers of different floral and geographic origins using ultrahigh-performance liquid chromatography-hybrid quadrupole-orbitrap mass spectrometry. J. Chromatogr. A 2017, 1499, 78-89. [CrossRef] [PubMed]

38. Chagas-Paula, D.; Zhang, T.; Da Costa, F.; Edrada-Ebel, R. A Metabolomic Approach to Target Compounds from the Asteraceae Family for Dual COX and LOX Inhibition. Metabolites 2015, 5, 404-430. [CrossRef] [PubMed] 